\title{
PROVIDING A WEB-BASED PLATFORM BASED ON PREDICTED WEIGHTS FROM EXISTING CONDITIONS
}

\author{
Navid Arandian, Saeed Behzadi \\ Shahid Rajaei University, Civil Engineer, Tehran, Iran, n.arandian.i@gmail.com \\ Shahid Rajaei University, Civil Engineer, Tehran, Iran, behzadi.saeed@gmail.com
}

KEY WORDS: Shortest path, web GIS, network analysis, neural network, Artificial intelligence

\begin{abstract}
Nowadays traffic problem has become a major dilemma due to the expansion of urbanization and the development of transportation. Traffic itself is the result of various factors, which can have an impact on the environment, and it has also destructive effects on human living. Finding a suitable method to reduce the negative effect of traffic has always been the subject of research in this area. Accordingly, there are different algorithms management and administrative procedures for solving this problem.

In this research, a web-based platform is designed using artificial intelligence algorithms, which predicts traffic information in different intervals along with online collecting traffic data. This feature allows the user to instantly view future information of urban traffic. On the other hand, in the proposed model, the transport network edges are determined based on traffic prediction algorithms, which makes route finding closer to reality.

The model is implemented on the 7th and 8th districts of Tehran. The algorithm has been applied to more than 100 cases and the results have been compared with existing algorithms. The results of this comparison show that in addition to higher precision, the proposed platform is averagely 10 minutes faster than similar programs.
\end{abstract}

\section{INTRODUCTION}

Traffic in the new era is one of the most important problems in modern society that has caused many problems. Traffic problems have always been one of the concerns of experts and experts in the field. Traffic has a negative impact on the environment and air pollution. It can also change the behavior of citizens, as far as it has become a psychological disaster. Accordingly, this great dilemma affects the psyche of individuals. Researchers found that even traffic - based sound pollution is one of the most important health threats. Traffic also increases blood pressure, heart attack and risk of diabetes. However, experts in urban management area believe in addition to training citizens and cultural change in the type of traffic, including:

1. Reinforcement of transport infrastructure

2. Creating a ratio of road capacity to business establishment points and recreational

3. Elimination of traffic congestion and geometric shapes of the pathways.
4. One way to get some passages in town can help reduce traffic.

However, one of the main objectives of this research is to obtain a structure that can store traffic data and generate the necessary traffic information for modeling. This structure can help us to reduce the traffic effects mentioned above. The issue of traffic in the past has been followed by researchers at different parts of the world. For example, the Traffic Analysis for Standards Association for Standards Association Using Learning, which is written by Naoki Hashimotoa, Seiichi Ozawaa, Tao Banb, Junji Nakazatob, kobe university [1], using the interdependence of the Traffic Association and the Internet has dealt with Traffic Analysis Traffic Analysis. It is also found in an article on traffic prediction in smart, which is by Attila M. In order to improve the quality and quantitative level of a smart city with modeling that has been done, Simon, of Technology 's 
Services and Services, University of Technology and Technology Services[2], has been forecast to predict future traffic conditions.

In this thesis, we try to introduce a new approach based on the analysis and advanced modeling algorithms in order to help reduce traffic. This starts with the storage of online and accuratetraffic data. Then using the existing algorithms the prediction of traffic conditions in the future pays. The more accurate the data available, is the more accurate of prediction.

\section{STUDIES AREA}

Tehran is the capital of Iran. Traffic is the biggest problem in the city. Many people are suffering from traffic in the city. So far, various measures have been taken to manage traffic in Tehran so that it can solve this problem, but no one has managed to solve the problem. the areas studied were the seventh and 8th places in tehran. tehran municipality is located in the capital city of tehran. It has five sections and 16 districts and an area of 15335212 sq. m. the city of tehran is also one of the urban areas of tehran located east of the city. It has three regions and 20 neighborhoods with an area of 13220000 sq. m.

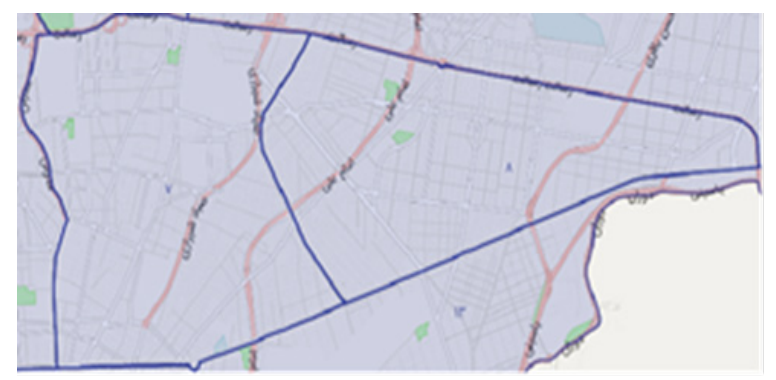

Figure 1: Tehran Municipality 7 and 8

\section{DATA}

\subsection{Design the system for traffic}

The main and most important data required in this research is traffic information. This information is generated by a number of companies, including the foreign samples, and the local sample of that site. the survey data has been used in this research, which provides the user with one of the most accurate types of data. The address of this site is https://www.mapquest.com. future. However, auxiliary can be done to reduce traffic.

This study consists of three main sections, which in the first part, the introduction, has given you an overview of the work and expression of the research literature. An explanation of the method is discussed in the following section. What the general method of this research has been and what steps have been made. Finally, with regard to the outcome of the work, we conclude that this work is done[3].

In this thesis, we try to design a system for traffic representation it is designed in a web environment. in order to do this, an online traffic layer, generally available by various services, has been evoked by the page on its screen. This layer actually possesses the traffic information of the streets in most countries in the world.

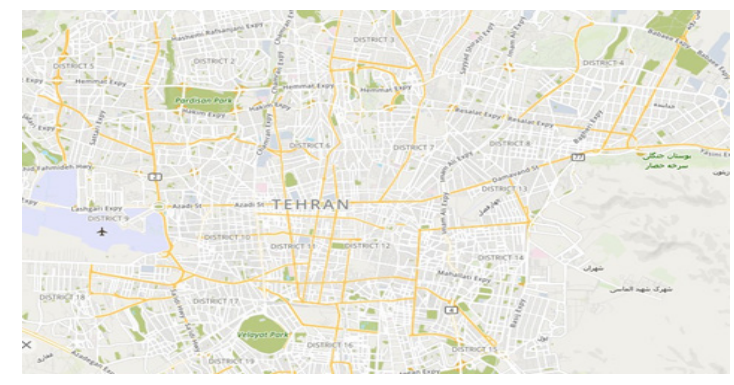

Figure 2: Part of the city's traffic in Tehran

3.2 Save the streets of the streets in the database and convert them to pixels

The second data required in this research is street information. Each of the streets has its own traffic, it has to construct a structure to save traffic information from each street separately. The database has been built in the face of the table. Given that each of the streets has a d of its own, we have saved these $\mathrm{d}$ for each of the streets separately in the database[4]. finally, in order to be able to read traffic information on each street, we have converted it separately to the point. This will enable the information to be stored in a single street. 


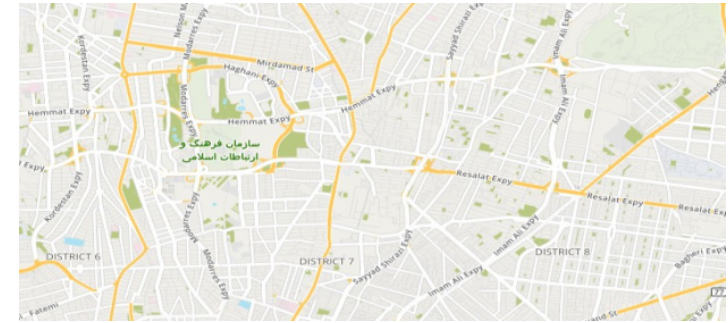

Figure 3: Saving streets Traffic Information

\section{METHODS}

4.1 Extracting the traffic from time to time and storing it in the database.

In this study, we first collect traffic data from the traffic layer every 5 minutes and is stored in the database. In fact, every five minutes of the page is automatically Refresh. Then, at any point in the Pin street, traffic information is derived from traffic as a color, including green traffic, which means low traffic, red in the sense of high traffic and black in the sense of traffic[5]. With the high number of traffic points and also their storage, we can measure the accuracy of the job.

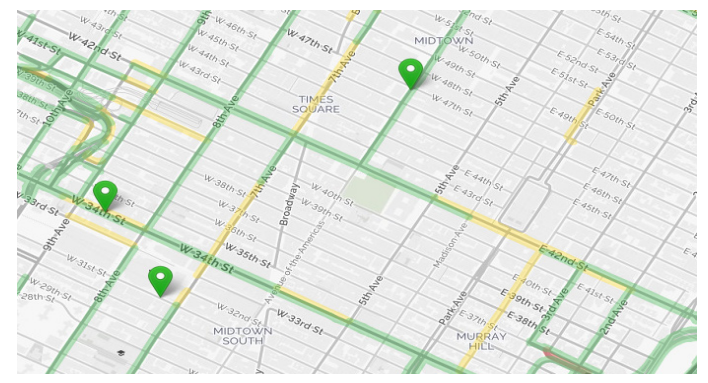

Figure 4: Streets traffic for a moment.

\subsection{Gather information for a specified range}

In order to predict the desired quality of the future traffic conditions in the future, such a more robust modeling is needed, so the need for a solid database is needed. Therefore, online traffic data have been stored in the database for a long time.

4.3 The implementation of the learning algorithm on the data collected and the prediction of the results.
Neural networks are modern systems and methods for machine learning, knowledge representation, and finally the practice of knowledge acquired in the direction of more optimism of output responses from complex systems. the main idea of these networks is partly inspired by the way biological neural system works to process data and information in order to learn and create knowledge. This system consists of a large number of highly interconnected processing elements called neurons, which act in harmony to solve a problem and transmit information via the synapses (electromagnetic connections).

An artificial neural network consists of three layers of input, output, and processing. Each layer consists of a group of neural cells ( neurons), commonly associated with all other layers of neurons, unless the user restricts the connection between the neurons ; but each layer is not connected to other layers of the same layer[6]. Using computer programming knowledge, data structure can be designed to function as a neuron. by creating a network of interconnected artificial neurons, the creation of an educational algorithm for the network and applying this algorithm to its network. these networks have a very high performance for approximation and approximation.

Given the above description, the position of the street and time as are given to the neural network. As described in the neural network function, the algorithm is implemented and the network creates a model. Finally, according to the type of function of the neural network, an output is generated, where information is related to the type of traffic in the street as our output. in the study, the results of the experiment have been obtained on over 100 cases.

\section{CONCLUSION}

As mentioned earlier, different communities suffer from traffic problems. in addition to the problems that arise in human time, it can cause a variety of diseases. Therefore, an attempt to reach solutions that will help reduce traffic is inevitable[7]. in this paper, we use a scientific approach to analyzing the traffic information and finally predicting the traffic conditions in the future. by using artificial intelligence algorithms, 
we were able to provide a solution to traffic reduction[8].

First, the region was chosen to conduct experiments and study on the cases where regions 7 and 8 of tehran were considered as studied area. To start the work at the first stage, we needed to collect certain traffic data that we meet from the www.mapquest.com site[9]. A system is then designed to display the information that can be understood visually. due to the existence of this information, there is no need to store this information. For this reason, we saved the traffic on the streets in five minutes. Given that each of the streets has its own traffic, we store each street separately and then at any point in the street traffic information is stored as a traffic layer color. In fact, each color represents the amount of traffic available. In a way that green is meant to mean traffic, yellow means high traffic, red in the sense of high traffic and traffic in the sense of traffic. To ensure that the accuracy of the job is high, this information is stored once every five minutes in a long period of time[10]. finally, due to the algorithm in artificial neural networks, the locations of the street and time were given to the algorithm and the traffic color output of each street was obtained. The benefits of this work, in addition to saving time for the user 's destination, can be referred to as traffic reduction, followed by the reduction of physical an.

\section{REFRENCES}

[1]. NaokiHashimoto, SeiichiOzawa, TaoBan, JunjiNakazato,JumpeiShimamura," A Darknet Traffic Analysis for IoT Malwares Using Association Rule Learning",INNS Conference on Big Data and Deep Learning 2018

[2]. Attila M.Nagy, VilmosSimon," Survey on traffic prediction in smart cities",Pervasive and Mobile Computing October 2018

[3]. Joshi Sujataa, Saxena Sakshamb, Godbole Tanvic, Shreyad," Developing Smart Cities: An Integrated Framework ",6th International Conference on Advances on Computing \& Communications, ICACC 2016, 6-8 September 2016, Cochin, India

[4]. Robertas Jucevipiusa, Irena Patašienơb, Martynas Patašiusc, "Digital dimension of smart city: critical analysis ", 19th International Scientific Conference; Economics and Management 2014, ICEM 2014, 2325April 2014, Riga, Latvia
[5]. Isabel Maria Lopesabc, Pedro Oliveiraa "Can a small city be considered a smart city? ", CENTERIS International Conference on ENTERprise Information Systems/ProjMAN-International Conference on Project MANagement/HCist -International Conference on Health and Social Care Information Systems and Technologies, CENTERIS /ProjMAN/ HCist 2017, 810 November 2017, Barcelona, Spain

[6]. Pierpaolo Girardi, Andrea Temporelli "Smartainability: a methodology for assessing the sustainability of the smart city " 8th International Conference on Sustainability in Energy and Buildings, SEB-16, 11-13 September 2016, Turin, ITALY

[7]. Tobias Brandt, Wolf Ketter, Lutz M. Kolbe,Dirk Neumann, Richard T. Watson "Smart Cities and Digitized Urban Management " Springer Fachmedien Wiesbaden $\mathrm{GmbH}$, part of Springer Nature 2018

[8]. Muhammad Babar, Fahim Arif, Mian Ahmad Jan, Zhiyuan Tan, Fazlullah Khan "Urban data management system: Towards Big Data analytics for internet Of things based smart urban environment using customized Hadoop " Future Generation Computer Systems September 2018

[9]. Trevor Braun, Benjamin C.M. Fung, Farkhund Iqbal, Babar Shah, "Security and Privacy Challenges in Smart Cities" (2018), https://doi.org/10.1016/j.scs.2018.02.039

[10]. Sriganesh K. Rao, Ramjee Prasad "Impact of 5G Technologies on Smart City Implementation" Springer Science+Business Media, LLC, part of Springer Nature 2018 